Editorial

\title{
Acknowledgement to Reviewers of Plants in 2019
}

Plants Editorial Office

MDPI, St. Alban-Anlage 66, 4052 Basel, Switzerland

Published: 17 January 2020

The editorial team greatly appreciates the reviewers who have dedicated their considerable time and expertise to the journal's rigorous editorial process over the past 12 months, regardless of whether the papers are finally published or not. In 2019, a total of 654 papers were published in the journal, with a median time to first decision of 14 days and a median time from submission to publication of 38 days. The editors would like to express their sincere gratitude to the following reviewers for their generous contribution in 2019:

Aalen, Reidunn Birgitta

Abbate, Loredana

Abdelrahman, Mostafa

Abdel-Tawab, Mona

Abe, Akira

Abinaya, Manivannan

Abraham, Eleni

Accoroni, Stefano

Achim, Cristina

Ács, Kamilla

Ádám, Attila L.

Adamakis, Ioannis-Dimosthenis

Adelberg, Jeffery

Adi, Kliot

Agathokleous, Evgenios

Agerbirk, Niels

Aguiar, Francisca C.

Ahmed, Mukhtar

Akhtar, Yasmin

Alam, Md. Khairul

Alamillo, Josefa Muñoz

Alba, Christina

Albreht, Alen

Aleksandrowicz-Trzcińska, Marta

Alexandrov, Oleg S.

Alexopoulos, Athanassios

Al-Fatimi, Mohamed

Alferez, Fernando

Alhousari, Fadi

Ali, Akhtar

Ali Zaidi, Shan

Alison M., Smith

Alkowni, Raed

Allen, Tom
Altemimi, AMMAR.B

Alvarez, Juan B.

Alves, Sheila

Alves, Tavvs M

Amarowicz, Ryszard

Ames, Ryan M.

Amirkhani, Masoume

Amsbury, Sam

An, Hong

Andolfi, Anna

Andrew, Tedder

Angelini, Riccardo

Anikó, Csecserits

Anower, Rokebul

Antal, Diana

Antoniadi, Ioanna

Antoniotti, Sylvain

Antoniou, Chrystalla

Antonkiewicz, Jacek

Anver, Shajahan

Aoki, Koh

Aparicio, Frederic

Appendino, Giovanni

Aranda, Ismael

Arcalis, Elsa

ArchMiller, Althea A.

Arena, Carmen

Ares, Ángela

Arévalo, José Ramón

Aroca, Angeles

Arora, Amandeep

Arturo Vera Ponce De Leon, Arturo

Artyszak, Arkadiusz

Aryal, Rishi 
Asard, Han

Ascencio-Ibanez, Trino

Asquith, Christopher R. M.

Astier, Jeremy

Astolfi, Stefania

Asztemborska, Monika

Athanasios, Dalakouras

Avellan, Astrid

Avesani, Linda

Avramidou, Evangelia

Avramova, Viktoriya

Axiotis, Evangelos

Azevedo, Herlander

Azzini, Elena

Bacelar, Eunice

Bąchor, Remigiusz

Backor, Martin

Badik, Kevin J.

Baek, You Soon

Bagheri, Hedayat

Bagniewska-Zadworna, Agnieszka

Bahmani, Ramin

Bai, Hua

Bailly, Christophe

Bajaj, Ruchika

Baker, C. Jacyn

Balasubramanian, Balamuralikrishnan

Baldoni, Elena

Balestrini, Raffaella Maria

Balslev, Henrik

Ban, Yusuke

Banach, Artur

Bandaralage, Jayeni Chathurika

Amarathunga Hiti

Banik, Chumki

Barberá, Gonzalo G.

Barcelo, Juan

Baritaki, Stavroula

Barnard-Kubow, Karen

Barreca, Davide

Barrios-Masias, Felipe $\mathrm{H}$.

Barrow, Chris J.

Barta, Csengele

Bartosz, Grzegorz

Baskin, Carol C.

Baslam, Marouane

Bassi, Daniele

Bassi, Roberto

Basu, Supratim

Baum, Christel

Bazghaleh, Navid

Bazos, Ioannis

Bazylko, Agnieszka
Bazzicalupo, Marco

Becana, Manuel

Beck, Andreas

Beckie, Hugh J.

Beers, Eric

Begara-Morales, Juan C.

Begcy, Kevin

Behrends, Volker

Beilby, Mary

Beissinger, Timothy

Bellairs, Sean

Bellaloui, Nacer

Bellucci, Michele

Benavides-Mendoza, Adalberto

Bencivenga, Stefano

Bender, Jurgen

Bender, Kyle

Benfey, Philip N

Beng Kah, Song

Benito, Begoña

Berberich, Thomas

Berendzen, Kenneth W.

BERGER, Antoine

Berger, Susanne

Bergero, Roberta

Berhow, Mark A.

Berim, Anna

Berlanga, Ángel Mérida

Berrocal-Lobo, Marta

Bervillé, André

Betekhtin, Alexander

Betti, Marco

Bhusal, Siddhi J.

Biagi, Marco

Białoń, Marietta

Białowiec, Andrzej

Bianco, Armandodoriano

Bicknell, Ross

Bidel, L.P.R.

Biligetu, Bill

Bilska-Kos, Anna

Biniari, Katerina

Biondi, Stefania

Biselli, Chiara

Bishopp, Anthony

Blanco, Antonio

Blanco-Salas, José

Blaylock, Michael J.

Bleeker, Petra M.

Blein, Thomas

Blein-Nicolas, Mélisande

Boccacci, Paolo

Bocianowski, Jan 
Boczkowska, Maja

Bogdan, Ileana

Bohanec, Borut

Boisivon, Helene Robert

Boissinot, Sylvaine

Bölcskei, Hedvig

Boldura, Oana-Maria

Bolibok-Braqgoszewska, Hanna

Bolle, Cordelia

Bombarely, Aureliano

Bonciu, Elena

Bonello, Pierluigi

Bonetta, Dario

Bonfil, David

Borek, Sławomir

Boris, Pejin

Boscaiu, Monica

Bostan, Hamed

Botton, Alessandro

Boudsocq, Marie

Bouhaouel, Imen

Bouranis, Dimitris L.

Bourque, Stéphane

Bouyahya, Abdelhakim

Boyce, Richard

Bozzo, Gale G.

Braam, Janet

Braglia, Luca

Brandt, Kirsten

Breidenbach, Natalie

Bresta, Panagiota

Brestic, Marian

Brew-Appiah, Rhoda

Brewin, Nick

Brighenti, Virginia

Brito, Cátia

Brogden, Kim

Bromfield, Elizabeth

Brooks, Peter

Browse, John

Brukhin, Vladimir

Bru-Martinez, Roque

Brunetti, Cecilia

Brunkard, Jacob O

Brusslan, Judy

Bubici, Giovanni

Buchholz, Anke

Budar, Françoise

Bueso, Eduardo

Bulgakov, Victor

Bulli, Peter

Bunce, James

Burghardt, Kyle
Burkey, Kent

Burrows, Geoffrey E.

Busby, Ryan R.

Butardo, Vito

Cabot, Catalina

Cacciola, Santa Olga

Cádiz-Gurrea, María De La Luz

Caeiro, Maria

Caetano, Nídia

Cahlíková, Lucie

Cahoon, A. Bruce

Cai, Giampiero

Calcutt, Michael

Calha, Isabel

Calín-Sánchez, Ángel

Callahan, Damien L.

Callis, Judy

Calvano, Cosima Damiana

CALZARANO, Francesco

Camacho-Cristóbal, Juan J.

Camañes, Gemma

Cámara-Leret, Rodrigo

Cámara-Zapata, José María

Campbell, Malachy T.

Campbell, Michael

Campbell, Shane

Cañas, Rafael A.

Candido, Vincenzo

Cândido, Elizabete

Caneva, Giulia

Canini, Antonella

Cánovas, Francisco

Cantamessa, Simone

Cao, Zhe

Capel, Carmen

Caputo, Lucia

Caracciolo, Giuseppina

Caradonia, Federica

Cardarelli, Mariateresa

Cardinale, Francesca

Cardoso, Hélia

Cardoso, Susana

Carella, Philip

Carillo, Petronia

Carocho, Márcio Soares

Carol, Pierre

Carpin, Sabine

Carrasco, Pedro

Carroll, Charles J.W.

Carrubba, Alessandra

Carullo, Gabriele

Caruso, Gianluca

Carvajal, Micaela 
Carvalho, Ana Maria Pinto

Casas, Ana M.

Casciaro, Bruno

Caser, Matteo

Castilho, Alexandra

Castilho, Paula C.

Castillo, Jesús

Castle, Alan

Castric, Vincent

Castro, Pedro Humberto

Castro-Vargas, Henry I.

Cavaco, Ana M.

Cazzato, Eugenio

Celiński, Konrad

Celletti, Silvia

Černý, Martin

Cervantes, Emilio

Chagne, David

Champion, Cody

Chandrasekaran, Murugesan

Chang, Wen-Chi

Chang, Yu-Sen

Chao, Louis Kuoping

Chao, Wen-Wan

Chapara, Venkat

Chapman, Mark

Chardon, Fabien

Charfeddine, Safa

Chen, Jen-Tsung

Chen, Po-Yuan

Chen, Xun

Chen, Yinglong

Cheng, Yuan-Bin

Cheon, Choong-ill

Cheung, Alice

Chibbar, Ravindra

Chin, Joong Hyoun

Chini, Andrea

Chitarra, Walter

Chiurazzi, Maurizio

Chlichlia, Katerina

Chmara, Rafał

Chmura, Damian

Cho, Man Ho

Chorostecki, Uciel

Christoffers, Michael

Chrysargyris, Antonios

Chrysoula, Tananaki

Chun, Sechul

Cilas, Christian

Cillo, Fabrizio

Cirvilleri, Gabriella

Clark, Gary
Clark, Gregory

Cober, Elroy

Cocozza, Claudia

Coelho, Livia

Cogato, Alessia

Cohen, Yigal R.

Cole, Logan W.

Collet, Trudi

Collino, Massimo

Combier, Jean-philippe

Cominelli, Eleonora

Commisso, Mauro

Conti, Heather

Conway, Warren

Cooke, Julia

Coppi, Andrea

Cordeiro, Nereida

Corpas, Javier

Correia, Carlos M.

Correia, Pedro

Cosoveanu, Andreea

Costa, Fabrizio

Costello, Ben De Lacy

Cox, Robert

Cozzolino, Daniel

Cretu, Mirela

Crevillén, Pedro

Crișan, Gianina

Crisp, Peter

Cruz, Cristina

Cséplő, Ágnes

Cvrčková, Helena

Czerwinska, Monika

Czerwińska, Monika

Da Silva, Jorge Marques

Dagnon, Soleya

D'Agostino, Nunzio

Dal Santo, Silvia

Damalas, Christos A.

DANG, THU-THUY

Danso-Boateng, Eric

Daras, Gerasimos

Dasgupta, Kasturi

Datta, Rahul

Dávalos, Andrea

David, Kopecky

David, Laure

David, Luminita

Davies, Peter J.

De Andrade, Alexander

De Bellis, Luigi

De Diego, Nuria

De Graaf, Barend HJ 
De Masi, Luigi

De Mieri, Maria

De Paolis, Angelo

De Tullio, Mario C.

De Vendittis, Emmanuele

De VIENNE, Dominique

De Vries, Jan

Debnath, Samir

Deckert, Joanna

Dehghan, Esmaeil

Del Genio, Charo

del Pozo, J. Carlos

Del Río Celestino, Mercedes

Delgado, Juan Antonio

Delgado, Sergio Nogales

Dello Ioio, Raffaele

Denholm, Ian

Deram, Annabelle

Dermastia, Marina

Deshmukh, Rupesh

Devireddy, Amith

Devlin, Paul

Dhandapani, Vignesh

Dharmasiri, Nihal

Dhiman, Saurabh

Di Bene, Claudia

Di Gioia, Francesco

Di Mambro, Riccardo

Di Martino, Catello

Di Mola, Ida

Di Rita, Federico

Di Sansebastiano, Gian-Pietro

Diaba, Faïza

Dietzgen, Ralf

DiGennaro, Peter

Dijk, Aalt

Dimitrakopoulos, Panayiotis G.

Ding, Baoqing

Ding, Jingyi

Dinis, Lia Tânia J

Dinkova, Tzvetanka D.

Diogène, Jorge

Divashuk, Mikhail

Djalali Farahani-Kofoet, Roxana

Djaman, Koffi

Dobrikova, Anelia

Docimo, Teresa

Dodd, Antony

Dodd, Ian

Doddapattar, Prakash

Doi, Kazuyuki

Dolatabadian, Aria

Dombrovsky, Aviv
Domozych, David S.

Dong, Yibo

Donno, Dario

Doria, Enrico

Dos Reis, André Rodrigues

DOSOKY, NOURA

Drabińska, Natalia

Drucker, Martin

Duan, Jialei

Duarte, Maria Cristina

Dubreucq, Bertrand

Dubrovina, Alexandra S.

Dugé De Bernonville, Thomas

Dun, Elizabeth

Duraisamy, Ganesh Selvaraj

Durgo, Ksenija

Dyderski, Marcin

Dyer, William

Dziadas, Mariusz

Eamens, Andrew L.

Ebert, Timothy A

Eckerstorfer, Michael

Edqvist, Johan

Edwards, Jeremy

Egamberdieva, Dilfuza

Egerton-Warburton, Louise

Eibl, Regine

Elameen, Abdelhameed

Elansary, Hosam O.

Eleftheriou, Eleftherios P.

El-Esawi, Mohamed

Elkins, Kelly

Elshamy, Abdelsamed

Endresen, Dag

Engelberth, Jurgen

Erel, Ran

Erktan, Amandine

Ermilova, Elena

Ersoz, Elhan S.

Ertani, Andrea

Ezer, Daphne

Falade, Titilayo D.O.

Falavigna, Vitor

Falistocco, Egizia

Fambrini, Marco

Fang, Francis

Fanizzi, Francesco Paolo

Fanourakis, Dimitrios

Faraone, Immacolata

Farcas, Anca Corina

Fares, Clara

Farzaneh, Vahid

Fascella, Giancarlo 
Fasoula, Vasilia

Fedderwitz, Frauke

Fehér, Attila

Fekete, Agnes

Felippes, Felipe Fenselau De

Feller, Urs

Fenesi, Annamária

Fernández, José A.

Fernández Barbero, Gerardo

Ferrante, Claudio

Ferrenberg, Scott

Ferrie, A. M. R.

Ferroni, Lorenzo

Festuccia, Nicola

Fetcher, Ned

Fialová, Silvia

Figaj, Donata

Figueroa-Bustos, Victoria

Fikere, Mulusew

Filip, Lorena

Fischer-Schrader, Katrin

Flakus, Adam

Flores García, Enrique

Florez-Sarasa, Igor

Foereid, Bente

Formenti, Ludovico

Forrer, Hans Rudolf

Forster, Britta

Forti, Luca

Fotopoulos, Vasileios

Francioso, Ornella

Franssen, Henk

Frolov, Andrej

Frugis, Giovanna

$\mathrm{Fu}$, Yong-Bi

Fujii, Hiroaki

Fujii, Sho

Fujimoto, Ryo

Fujita, Daisuke

Fujita, Masayuki

Fujiwara, Takayuki

Fujiyama, Hideyasu

Fukuda, Tomohiko

Fukui, Kenji

Fukunaga, Kenji

Fukushima, Atsushi

Fukushima, Kenji

Funada, Ryo

Fung Uceda, Jorge

Funnekotter, Bryn

Gabbia, Daniela

Gabrielli, Paolo

Gabryś, Beata
Gaggini, Luca

Galasiti Kankanamalage, Anushka

Galiba, Gabor

Gallardo-Guerrero, Karine

Gallego, Juan Carlos Alías

Gallemí, Marçal

Galoburda, Ruta

Galstyan, Anahit

Galvão, Vinicius Costa

Gambino, Giorgio

Gan, Susheng

Ganesan, Palanivel

Gangola, Manu Pratap

Ganič, Karin Kovačević

Gannon, Bryan

Garcia De Leon, David

García-Cervigón, Ana I

García-Estañ, Joaquín

Garcia-Sanchez, Francisco

Garmendia, Idoia

Garzoli, Stefania

Gasparatos, Dionisios

Gasperini, Debora

Gates, Colin

Gatz, Christiane

Gauslaa, Yngvar

Gavilan, Rosario

Gavilán, Rosario G.

Geisler, Matt

Gent, Martin

Genta-Jouve, Grégory

Georgiev, Vasil

Georgiev, Yordan

Gerke, Jörg

Germ, Mateja

Gervasi, Teresa

Gesell, Andreas

Ghanizadeh, Hossein

Ghatak, Arindam

Ghosal, Sambuddha

Ghose, Kaushik

Giacomello, Stefania

Gianinetti, Alberto

Gibson, David John

Gilardi, Giovanna

Gilding, Edward K.

Giles, Wayne

Giordani, Paolo

Giordano, Simonetta

Giritch, Anatoli

Giurg, Mirosław

Gladish, Daniel K.

Glibowski, Paweł 
Głowacka, Katarzyna

Godena, Sara

Goicoechea, Nieves

Goldfarb, Barry

Goldschmidt, Eliezer E.

Gollan, Peter J.

Golob, Aleksandra

Golubkina, Nadezhda

Gomes, Eric

Gómez Cadenas, Aurelio

Gomez Camacho, Carlos Enrique

Gomez Roldan, Maria Victoria

Gonçalves, Sandra

Gonda, Sandor

Gontier, Eric

González Andújar, José Luis

González-Domínguez, Raúl

Gorchov, David

Gori, Antonella

Gorres, Josef H.

Gospodarek, Janina

Gossman, Toni

Goyal, Etika

Grabelnych, Olga I.

Grace, John

Gradov, Oleg V.

Gradziel, Thomas M.

Grafi, Gideon

Gramzow, Lydia

Granica, Sebastian

Grantz, David

Grattan, Stephen

Greenberg, Anthony

Greenlief, C. Michael

Greer, Dennis

Gregersen, Per

Grey, Tim

Grones, Peter

Grote, Rüdiger

Groten, Karin

Grubišić, Dinka

Grul'ová, Daniela

Grumet, Rebecca

Grzebelus, Dariusz

Grzebelus, Ewa

Gu, Xingyou

Guadagno, Carmela

Gueguen, Erwan

Guerra-Sanchez, Guadalupe

Guerriero, Gea

Guevara, Ramón

Guevara González, Ramón

Guil-Guerrero, José Luis
Gujas, Bojan

Gulli, Mariolina

Guo, Hao-Bo

Guo, Zhanjun

Gupta, Parul

Gutekunst, Kirstin

Gutiérrez-Miceli, Federico

Guy, Charles L.

Guzmán-Delgado, Paula

H. Gonzalez, Daniel

Ha, Chien Van

Hădărugă, Nicoleta

Hagerty, Christina

Halassy, Melinda

Hamman, Sarah T.

Hammerschmidt, Ray

Hammond, John

Hamouzová, Kateřina

Han, Yeon Soo

Hanaka, Agnieszka

Hanania, Michel

Hanaoka, Mitsumasa

Hancock, John

Hano, Christophe

Hanus-Fajerska, Ewa

Hardion, Laurent

Harman, Gary

Harmens, Harry

Harrington, Kerry C.

Hartl, Markus

Hasanuzzaman, Mirza

Haseeb, Muhammad

Hasegawa, Paul Michael

Hasiów-Jaroszewska, Beata

Hassan, Sherif T. S.

Hatano, Tsutomu

Hatzpolous, Polydefkis

Hause, Bettina

Hawrylak-Nowak, Barbara

Hay, Angela

Hayashi, Satomi

Hayes, Patrick

Hearn, David

Heckenhauer, Jacqueline

Hedden, Peter

Heger, Tina

Hell, Rüdiger

Helliwell, Chris

Helm, Richard

Henry, Robert

Herath, Venura

Hernández, José A.

Herranz, Raúl 
Herrero, Baudilio

Herzberg, Martin

Hess, Michael

Hessel-Pras, Stefanie

Heyer, Arnd

Higgs, David

Hilliou, Frederique

Hinojosa, Leonardo

Hirakawa, Yoshihisa

Hirel, Bertrand

Hirotsu, Naoki

Hirschi, Kendal

Ho, Hon

Hodson, Martin

Hojsgaard, Diego

Holalu, Srinidhi

Holec, Josef

Hölzl, Georg

Hong, Yiguo

Horel, Ágota

Hori, Kiyosumi

Horiguchi, Gorou

Hortigón-Vinagre, María

Horvath, David

Hossein, Borhan

Hosseini, Seyed Abdollah

Howe, Gregg

Hsia, Shih-Min

Hsieh, Hsu-Liang

Hsu, Yi Ting

$\mathrm{Hu}$, Guanjing

Huang, Guan-Jhong

Huang, Lei

Huang, Shuanglong

Huang, Wen-Lii

Huang, Xingfeng

Hubert, Sytykiewicz

Huchzermeyer, Bernhard

Hura, Tomasz

Husbands, Aman

Hwang, Woon-Ha

Ian, Dundas

Ianole, Rodica

Iantcheva, Anelia

Iapichino, Giovanni

Ibba, Maria Itria

Ibrahim, Salam

Ientile, Riccardo

Igamberdiev, Abir U.

Ikeda, Hajime

Ilardi, Vincenza

Imamura, Sousuke

Inagaki, Noritoshi
Innangi, Michele

Iorizzi, Maria

Irga, Peter

Irish, Erin

Irving, Louis

Ishiguro, Sumie

Ishijima, Sumio

Ishikawa, Ryuji

Ishizaki, Takuma

Ishizuka, Wataru

Islam, Md. Tabibul

Islam, Mohammad Zahirul

Itabashi, Etsuko

Ito, Michiho

Ivanov, Alexander

Ivanov, Ivan

Iwakami, Satoshi

Iwata, Yuji

J. Barba, Francisco

Jabloun, Mohamed

Jain, Shri Mohan

Jakkula, Vinod

Jallet, Denis

Jan, Fuh-Jyh

Janda, Elzbieta

Jang, Seonghoe

Janga, Madhusudhana

Janicka-Russak, Małgorzata

Janssen, Dirk

Jaradat, Nidal

Jarolmasjed, Sanaz

Jarquin, Diego

Jarret, Robert L.

Jarugula, Sridhar

Jeandroz, Sylvain

Jellen, Eric

Jeong, Dong-Hoon

Jeong, Rae-Dong

Jerkowić, Igor

Jewell, Jeremy B.

Jhanwar, Shalu

Jiang, Shu-Ye

Jicsinszky, László

Jiménez Fernández, Alicia

Jimenez-Lopez, Jose Carlos

Jobe, Timothy

Jocković, Milan

Johnson, Donn

Johnson, Eric

Johnson, Giles

Jones, Meriel

Jones, Richard W

Jopcik, Martin 
Jordan-Meille, Lionel

Jordão, António Manuel

Jordheim, Monica

Jørgensen, Hans Jørgen Lyngs

Jorrin, Beatriz

Jose, Rodriguez

Juárez-Escario, Alejandro

Julien, Gronnier

Jullian, Nathalie

Jung, Ki-Hong

Juráň, Stanislav

Jurkonienè, Sigita

Kadela-Tomanek, Monika

Kafle, Lekhnath

KAINOH, Yooichi

Kaitaniemi, Pekka

Kakrana, Atul

Kalachova, Tetiana

Kalaji, Hazem Mohamed

Kalemba, Ewa M.

Kaliniewicz, Zdzisław

Kalyuzhnaya, Marina

Kaminaka, Hironori

Kanehara, Kazue

Kanissery, Ramdas G.

Kapantaidaki, Despoina Ev.

Karahara, Ichirou

Karcz, Dariusz

Karkanis, Anestis

Karmakar, Partha

Karoglan, Marko

Kartashov, Alexander

Kasahara, Ryushiro D.

Kato, Keita

Kato, Yoshio

Katsuhara, Maki

Kaur, Harmanjit

Kawade, Kensuke

Kawamura, Fumio

Kayama, Masazumi

Kazama, Tomohiko

Keereetaweep, Jantana

Keinänen, Markku

Kellogg, Joshua J.

Kenter, Christine

Kern, Ramona

Kerwin, Rachel E.

Kerwin, Sean M.

Khallouki, Farid

Khanal, Sameer

Khazaei, Hamid

Kheradpezhouh, Ehsan

Khosla, Aashima
Kibet, Leonard

Kikowska, Małgorzata

Kil, Eui-Joon

Kim, In-Jung

Kim, Jin-Hong

Kim, Jin-Kyung

Kim, Ki Hyun

Kim, Kyung-Min

Kim, Sukweon

Kim, Tae-Hwan

Kim, Woe-Yeon

Kim, Yong-Ick

Kimbaris, Athanasios

Kimura, Seisuke

Kinose, Yoshiyuki

Kinugasa, Toshihiko

Kiraly, Lorant

Kiselev, Konstantin V.

Klauser, Dominik

Klčová, Lenka

Kleczkowski, Leszek A.

Knoshaug, Eric

Ko, Kisung

Kobae, Yoshihiro

Kocábek, Tomáš

Kocira, Sławomir

Koga, Hiroyuki

Koide, Yohei

Koizuka, Nobuya

Koji, Mikami

Kolbert, Zsuzsanna

Kollárová, Karin

Kolodziejczyk, Joanna

Kolukisaoglu, Üner

Komenda, Josef

Komínková, Dana

Komis, George

Komis, George

Konar, Arkaprabha

Kong Thoo Lin, Paul

Konopka, Iwona

Konopka-Postupolska, Dorota

Kontunen-Soppela, Sari

Kooij, Pepijn W.

Korell, Lotte

Kosmala, Monika

Kosova, Klara

Kosterin, Oleg

Kotta-Loizou, Ioly

Kowalczyk, Mariusz

Kowalski, Radosław

Kozaki, Akiko

Kozieł, Edmund 
Kozlov, Mikhail V.

Kraj, Wojciech

Kramer, Elena M.

Krasniewska, Karolina

Kriauciuniene, Zita

Kriechbaumer, Verena

Krier, François

Krishnamurthy, Pannaga

Kroj, Thomas

Krüger, Stephan

Kuboyama, Tsutomu

Kucukoglu, Melis

Kudoyarova, Guzel

Kukal, Meetpal

Kulhánek, Martin

Kulich, Ivan

Kulkarni, Shashank

Kulma, Anna

Kulus, Dariusz

Kumar, Jitendra

Kumar, Manu

Kumar, Narender

Kumar Kundu, Jiban

Kunihisa, Miyuki

Kunwar, Ripu MARDHAN

Kuo, Yao-Haur

Kuta, Elżbieta

Kwiatkowski, Pawel

Kwon, Choon-Tak

Kwon, Suk-Yoon

La Spada, Federico

Laane, Henk-Maarten

Labate, Joanne

Labudda, Mateusz

Lachman, Jaromir

Lacis, Gunars

Lahuta, Lesław Bernard

Lam, Sio-Hong

Lamb, Dane T.

Landoni, Michela

Lange, Bastien

Langner, Thorsten

Lao Arenas, Maria Teresa

Laranjo, Marta

Larkan, Nicholas

Larkum, Anthony

Larson, Steven R.

Lastochkina, Oksana

László, Makra

Latowski, Dariusz

Laur, Joan

Lavelle, Dean O.

Lavelli, Vera
Lavin, Matt

Lazarević, Boris

Lazaro, Lauren

Le Hir, Rozenn

Lebedev, Vadim G.

Leborgne-Castel, Nathalie

Ledeunff, Erwan

Lee, Byung-Hoo

Lee, Jung Ro

Lee, Keunsub

Lee, Natuschka

Lehtonen, Samuli

Leitão, José M.

Leiva-Brondo, Miguel

Lemos, Rafael Plá Matielo

Lentini, Giovanni

Leon-González, Antonio J.

León-Rivera, Ismael

Lepeduš, Hrvoje

Leru, Poliana

Leszczuk, Agata

Levitsky, Victor G.

Leyva Pérez, Maria De La O

Li, Po-Hsien

Li, Qiansheng

Li, Wanlong

Li, Xiaoguang

Li, Yin

Li, Zhongyi

Li Destri Nicosia, Maria Giulia

Liang, Zhikai

Liao, Yuxing

Lico, Chiara

Lidon, Fernando

Ligaba-Osena, Ayalew

Liguori, Giorgia

Lima-Brito, José

Lin, Hung-Yin

Lin, Tung-Yi

Lin, Wei-Yi

Ling, Maurice

Linhari Rodrigues Pietro, Rosemeire Cristina

Linkies, Ada

Lionetti, Vincenzo

Liu, Haipei

Liu, Ke

Liu, Lei

Liu, Liyun

Liu, Ming-Jung

Liu, Qing

Liu, Shao-Lun (Allen)

Liu, Sijun

Liu, Weizhen 
Liu, Yongliang

Livanos, Pantelis

Lizamore, Darrell

Llorens, Eugenio

Loake, Gary

Lognay, Georges

Logozzo, Giuseppina

Loizzo, Monica R.

Loka, Dimitra

Lomonaco, Tommaso

Lopes, Graciliana

López Saéz, José Antonio

Loppi, Stefano

Lora, Jorge

Lord, Janice

Lordan, Ronan

Lorenz, Peter

Łotocka, Barbara

Løvdal, Trond

Loveridge, Joel

Lovisolo, Claudio

Lozano-Juste, Jorge

$\mathrm{Lu}, \mathrm{Chi}-\mathrm{Yu}$

$\mathrm{Lu}$, You

Lucas, Jose Antonio

Lucek, Kay

Lucena, Carlos

Lucioli, Simona

Lukhovitskaya, Nina

Lullien, Valerie

Lunardon, Alice

Lutter, Petra

Lüttge, Ulrich E.

Luxova, Miroslava

Łyczakowski, Jan

Ma, Guojia

Maag, Daniel

Mabou Tagne, Alex

Macfie, Sheila M.

Maggi, Filippo

Maghuly, Fatemeh

Magnano Di San Lio, Gaetano

Mahdavi Mazdeh, Parastoo

Maier, Camelia

Maizel, Alexis

Malacrinò, Antonino

Malla, Subas

Malladi, Anish

Mallory-Smith, Carol

Malyan, Sandeep

Manfredini, Stefano

Mang, Stefania Mirela

Mano, Jun'ichi
Mantovani, Roberto

Mantzouridou, Fani

Marasek-Ciolakowska, Agnieszka

Marček, Tihana

Marcel, Sylvain

Marchand, Patrice A.

Marchi, Maurizio

Marcon, Caroline

Mareri, Lavinia

Marhuenda, Javier

Marincas, Olivian

Marino, Daniel

Markowicz-Piasecka, Magdalena

Marra, Roberta

Marrelli, Mariangela

Marsol, Alexis

Martí, Raúl

Martinez, Manuel

Martinez, Shantel

Martínez, Sidonia

Martinez Ruiz, Carolina

Martínez-Gómez, Pedro

Martínez-Sánchez, Gregorio

Martínez-Turiño, Sandra

Martino, Elena

Martins, Alice M.

Martins, Isabel

Martins-Loução, Maria Amélia

Martz, Francoise

Maruta, Takanori

Maruyama-Nakashita, Akiko

Maserti, Bianca Elena

Mashima, Ryuichi

Masin, Roberta

Masson, Patrick H.

Mastanjević, Krešimir

Masuda, Sachiko

Masuda, Tatsuru

Mata-Pérez, Capilla

Mateo-Bonmatí, Eduardo

Matha, Shivaraj

Mathesius, Ulrike

Matichenkov, Vladimir

Matilla, Angel J.

Matilla, Miguel

Matsubara, Kazuki

Matsumoto, Takahiro

Matthes, Annemarie

Mattoo, Autar

Mattsson, Jim

Matuszewska, Anna

Matzrafi, Maor

Maulana, Frank 
Mayes, Sean

Mayton, Hilary

McFarlane, Heather E.

McInroy, John A.

Mcintosh, Robert

McKenzie, Marian Jane

McKeown, Peter C.

McNeil, David

Means, Nathan

Medina, Francisco Javier

Medo, Juraj

Meeks, Calvin

Mehariya, Sanjeet

Mei, Yang

Meisrimler, Claudia-Nicole

Meki, Norman

Meletiou-Christou, Maria-Sonia

Melosik, Iwona

Meneghetti, Fiorella

Meneguzzo, Francesco

Menendez, Esther

Menet, Marie Claude

Mens, Celine

Mensuali-Sodi, Anna

Merah, Othmane

Merai, Zsuzsanna

Mercati, Francesco

Merino, Irene

Mészáros, Ilona

Meurer, Jörg

Mezzetti, Bruno

M-Hamvas, Márta

Miao, Chenyong

Michalak, Marcin

Michaud, Morgane

Michels, Alexander J.

Mierek-Adamska, Agnieszka

Mierek-Adamska, Agnieszka

Milan, Marco

Minocha, Subhash

Mishra, Amit Kumar

Mithoefer, Axel

Mittapelly, Priyanka

Miyamoto, Koji

Mizuno, Takayuki

Mogoșan, Cristina

Mohamed, Azza H.

Moir, Jim

Molin, William

Molinier, Jean

Møller, Ian Max

Monneveux, Philippe

Monteiro, Filipa
Montes, Nuria

Moolhuijzen, Paula M.

Moon, Kyung

Moore, Nathan

Moraes, Thiago

Moraga, Javier

Morales, Laura

Morales-Quintana, Luis

Morandi, Brunella

Moravcikova, Jana

Morello, Laura

Moreno, Jordi

Morgan, John

Mori, Kazuhiro

Moricz, Agnes

Moriguchi, Takaya

Morita, Shigeto

Morra, Matthew J.

Morris, Paul Francis

Morrow, Jennifer

Moschou, Panagiotis N.

Mota, Manuel

Mottaghipisheh, Javad

Motte, Hans

Mourato, Miguel P.

Mucha, Joanna

Mulas, Maurizio

Muleo, Rosario

Mundy, D.C.

Muñoz, Alfonso

Muñoz-Centeno, Luz María

Murillo, Pablo García

Mustroph, Angelika

Muszyńska, Ewa

Myrta, Arben

Nadakuduti, Satya Swathi

Nadeem, Muhammad

Nadgórska-Socha, Aleksandra

Nagahage, Isura

Nagashima, Yukihiro

Nagy, Istvan

Nakajima, Masami

Nakamura, Yuki

Nakano, Michiharu

Nakashima, Souichi

Nallu, Sumitha

Namba, Shigetou

Napoli, Marco

Nardi, Serenella

Nardini, Andrea

Nardozza, Simona

Narouei-Khandan, Hossein

Naumovski, Nenad 
Navarro-Alarcon, Miguel

Nawade, Bhagwat

Nawaz, Muhammad Amjad

Neji, Mohamed

Nelson, Matthew

Nemeskéri, Eszter

Neng, Nuno

Neri, Davide

Neufeld, Howard

Neupane, Achal

Newcombe, George

Newman, Mari-Anne

Nicosia, Aldo

Niederman, Robert A.

Nielsen, Brent

Niemczyk, Marzena

Nieto, Gema

Nilsen, Erik T

Nin, Stefania

Niu, Genhua

Nogales, Amaia

Noguchi, Ko

Nogues, Isabel

Nonhebel, Heather M.

Noordergraaf, Inge-Willem

Noris, Emanuela

Nosalewicz, Artur

Novikov, Andriy

Novikov, Arthur I.

Novo, Luís

Nürnberg, Dennis

Nybakken, Line

Nybom, Hilde

O. Miller, Jarrod

Ocamb, Cynthia M.

Ofir, Rivka

Ogata, Yoshiyuki

Ogórek, Rafał

Ogunjirin, Adebowale

Oh, Seung-Yoon

Ohtomo, Ryo

Ohtsu, Naoko

Ohyama, Takuji

Okamura, Yu

Okazawa, Atsushi

Oklestkova, Jana

Okorski, Adam

Okubara, Patricia

O'Leary, Brendan M.

Olejar, Kenneth J.

Olmos, Antonio

Olmos, Enrique

Olsson, Sanna
Omar, Ahmad

Omidbakhsh-Fard, M. Amin

Ondreičková, Katarína

Ondrej, Vladan

Ong, Eng Shi

Oniga, Ilioara

Oniszczuk, Anna

Öpik, Maarja

Ordas, Bernardo

Orlova-Bienkowskaja, Marina J.

O'Rourke, Jamie

Ortega, John

Ortiz-Urquiza, Almudena

Ostrowski, Maciej

Oszako, Tomasz

Otagaki, Shungo

Otulak-Kozieł, Katarzyna

$\mathrm{Ou}$, Junjun

Ougham, Helen

Ouzounidou, Georgia

Overmyer, Kirk

Owens, Daniel K.

Ozaki, Noriaki

Ozimek, Ewa

Pacuta, Vladimir

Pagán, Israel

Pagliarani, Chiara

Pagnotta, Mario A.

Paixao Coelho, Jose Augusto

Pająk, Paulina

Pál, Magda

Palacios, José-Manuel

Palacios, Sara

Palanivelu, Ravi

Palla, Franco

Palomo Ríos, Elena

Palove Balang, Peter

Palumbo, Fabio

Panara, Francesco

Pandolfini, Tiziana

Panek, Jacek

Papageorgiou, Aristotelis

Papatheodorou, Efimia M.

Papini, Alessio

Paponov, Ivan A.

Pappa, Aglaia

Pappas, Christos

Paradjikovic, Nada

Parajuli, Saroj

Park, Chankyu

Park, Il-Kwon

Park, Inkyu

Park, Sang-Wook 
Park, Sunghun

Parker, Steven L.

Pârvu, Alina Elena

Pasternak, Taras

Patil, Abhinandan

Paul, Matthew

Paul, Narayan Chandra

Pavela, Roman

Pazzagli, Luigia

Pečenková, Tamara

Pedersen, Henrik

Pedrazzini, Emanuela

Pedroza-Garcia, Jose-Antonio

Peirats-Llobet, Marta

Peixe, Augusto

Pék, Zoltán

Peltomaa, Elina

Peña-Gómez, Nataly

Peng, Ze

Perdomo, Juan Alejandro

Pereira, Paula Natália

Pereira-Lorenzo, Santiago

Perez, Marta

Perez De Souza, Leonardo

Pérez-Fernández, María

Pernisová, Markéta

Pernot, Clémentine

Perotto, Silvia

Perrine-Walker, Francine

Perry, Sharyn

Perucka, Irena

Peška, Vratislav

Petek, Marko

Petoumenou, Despoina G.

Petricevich, Vera

Petrivalsky, Marek

Petropoulos, Spyridon

Petrussa, Elisa

Petti, Carloalberto

Peyambari, Mahtab

Pfister, Barbara

Piasecki, Cristiano

Piccionello, Antonio Palumbo

Piccolella, Simona

Pickard, Barbara

Piechulla, Birgit

Piedras, Pedro

Piemontese, Luca

Piepenbring, Meike

Piepho, Hans-Peter

Pietrowska-Borek, Małgorzata

Piispanen, Riikka

Pilu, Roberto
Pingault, Lise

Pinker, Ina

Pitingolo, Gabriele

Piya, Sarbottam

Pizzeghello, Diego

Plader, Wojciech

Platta, Harald

PŁażek, Agnieszka

Pleskot, Roman

Plíhal, Ondřej

Plíhalová, Lucie

Pniewski, Tomasz

Pokluda, Robert

Polidoros, Alexios

Pollastro, Stefania

Pongrac, Paula

Ponte, Nuno

Ponz, Fernando

Poór, Péter

Popova, Antoaneta

Porceddu, Marco

Potrykus, Marta

Poudyal, Shital

Pourret, Olivier

Pozzi, Cecilia

Prado-Cabrero, Alfonso

Pramod, Sreepriya

Pranckeviciene, Erinija

Prasad, Kasavajhala V. S. K.

Prasad, Ram

Prashar, Sanjiv

Premachandra, Gnanasiri S.

Pretel, Maria Teresa

Price, Elliott James

Pringle, Elizabeth

Prinsen, Els

Prudent, Marion

Pucci, Nicoletta

Puccinelli, Martina

Pucker, Boas

Puglia, Giuseppe

Pugliesi, Claudio

Puglisi, Ivana

Pugovics, Osvalds

Puig, Carolina G.

Qaderi, Mirwais

Qiu, Huan

Quambusch, Mona

Querner, Pascal

Quesada Pérez, Víctor

Race, Marco

Radford, Ian J.

Radić, Tomislav 
Rahi, Lifat

Rahman, Md. Atikur

Raikhy, Gaurav

Rajarapu, Swapna Priya

Rallo, Luis

Ramana Pidatala, Venkata

Ramegowda, Venkategowda

Ramesh, Sunita A.

Ramos-Solano, Beatriz

Rapeanu, Gabriela

Rascio, Agata

Rasmussen, Soren

Rass, Ulrich

Rastogi, Anshu

Ratcliffe, Richard George

Raudabaugh, Daniel

Raudonè, Lina

Ravelonandro, Michel

Ravin, Nikolai V.

Ravlić, Marija

Rayon, Catherine

Reda, Małgorzata

Reddy Janga, Madhusudana

Reed, Kevin F.M.

Reglinski, Tony

Regni, Luca

Rehman, Junaid Ur

Rehrig, Erin M.

Reig, Gemma

Reigosa, Roger J.

Ren, Hong

Renault, Sylvie

Renna, Massimiliano

Resentini, Francesca

Restuccia, Alessia

Revuelta, Julia

Rezzani, Rita

Rho, Hyungmin

Rial, Carlos

Ricci, Antonella

Richard, Benjamin

Richter, René

Rigas, Stamatis

Rigó, Gábor

Rigoldi, Maria Pia

Rischer, Heiko

Rivero, Rosa M

Roach, Thomas

Robert, Elisabeth M. R.

Robin, Arif Hasan Khan

Roche, Benjamin

Roden, John

Rodrigo, Joaquin
Rodrigues, António S.

Rodrigues, Célia F.

Rodríguez, Víctor Manuel

Rodríguez De Rivera, Óscar

Rodríguez Villanueva, Javier

Rodríguez-González, Álvaro

Rodríguez-Merino, Argantonio

Rodríguez-Peña, Rosa A.

Rodríguez-Soalleiro, Roque

Rodríguez-Yoldi, María Jesús

Rogers, Hilary

Rogozhin, Eugene

Rohila, Jai

Rollin, Patrick

Romeiras, Maria M.

Romeiras, Maria Manuel

Romero, Irene

Romero, José

Romero-Puertas, María C.

Romuli, Sebastian

Ronga, Domenico

Roni, Md Zohurul Kadir

Ros, Roc

Roscini, Luca

Rosellini, Daniele

Rossato, Marzia

Roubelakis-Angelakis, Kalliopi A.

Routray, Winny

Royo-Esnal, Aritz

Royuela, Mercedes

Rozhon, Wilfried

Rozov, Serge M.

Różyło, Krzysztof

Rudnóy, Szabolcs

Rudrabhatla, Sairam

Rudzińska, Magdalena

Ruelland, Eric

Ruggeri, Roberto

Rugini, Eddo

Ruiz Talonia, Lorena

Ruiz Téllez, Trinidad

Runions, Adam

Rurek, Michal

Russell, Scott

Russo, Daniela

Rutto, Laban

Rybczyńska-Tkaczyk, Kamila

Rybka, Krystyna

Saarto, Annika

Sabater, Bartolome

Sabella, Erika

Sadot, Einat

Saha, Sushanta Kumar 
Sahab, Sareena

Sahrawy, Mariam

Saiano, Filippo

Saitanis, Costantinos

Saito, Yoshiro

Sakamoto, Tomoaki

Sakar, Germán

Sakata, Yuzu

Sakkas, Hercules

Salachna, Piotr

Salas, José Blanco

Salmerón-Sánchez, Esteban

Salome, Patrice

Salopek-Sondi, Branka

Samach, Alon

Šamec, Dunja

Sanchez, Darlene

Sánchez Torres, Paloma

Sandalio, Luisa María

Sangireddy, Sasikiran Reddy

Sansone, Anna

Santino, Angelo

Santos, Ana Paula

Santos, Bruno A C M

Santos, Carla S.

Santos, Rita B.

Santos, Susana

Sanz-Alférez, Soledad

Sanz-Saez, Alvaro

Sara, Montanari

Sarkar, Abhijit

Sarkar, Saswata Sankar

Satish, Lakkakula

Sato, Hikaru

Sato, Norihiro

Sattar, Sampurna

Sauer, Michael

Savchenko, Tatyana V.

Savoi, Stefania

Sawicki, Rafal

Scala, Valeria

Scalabrin, Elisa

Scandellari, Francesca

Scarafoni, Alessio

Scavo, Aurelio

Schaffer, Arthur A.

Schaller, Jörg

Schaub, Marcus

Schiavon, Michela

Schlüter, Urte

Schmeda-Hirschmann, Guillermo

Schmidt, Lilian

Schmidt, Martin
Schmitt-Keichinger, Corinne

Schoenaers, Sébastjen

Schreier, Tina

Schroeder, Hilke

Schubert, Marian

Schulz, Alexander

Schulze, Stefanie

Schuster, Gadi

Schuster, Michael

Scognamiglio, Monica

Scorziello, Antonella

Scotti, Nunzia

Sebastiana, Mónica

Sebastiani, Federico

Seca, Ana Maria Loureiro Da

Segura, Ana

Seifert, Georg J.

Seo, Eunyoung

Serafini, Mauro

Serafini-Fracasini, Danatella

SERALINI, Gilles-Eric

Sergeant, Kjell

Serrano, María

Setamou, Mamoudou

Seto, Shigeki

Setzer, William N.

Seung, David

Sevilla-Morán, Beatriz

Sgorbini, Barbara

Shabbir, Asad

Shafiq, Sarfraz

Shahrokhnia, Hossein

Shahzad, Babar

Shaikhali, Jehad

Sharma, Harmandeep

Sharma, Manisha

Sharpe, Richard M.

Shen, JIANQIANG

Sheng, Ruilong

Shi, Xiaowen

Shibuya, Kenichi

Shim, Donghwan

Shimada, Tomoo

Shimojima, Mie

Shiratani, Masaharu

Shishova, Maria F.

Shrestha, Krishna

Shukla, Mukund

Shymanovich, Tatsiana

Siatkowski, Idzi

Sidhu, Gaganjot

Sidhu, Virinder

Sieber, Alisa-Naomi 
Silar, Philippe

Šiler, Branislav

Silva Dias, Luís

Silvestri, Cristian

Simkin, Andrew

Simm, Stefan

Sims, Brian

Singh, Ashutosh

Singh, Manjul

Singh, Narinder

Singh, Reema

Sipes, Brent

Sivanesan, Iyyakkannu

Skaracis, George

Skendi, Adriana

Skjelvåg, Arne Oddvar

Skorko-Glonek, Joanna

Skrypnik, Liubov N.

Ślipiko, Monika

Śliwińska-Wilczewska, Sylwia

Sloan, Daniel

Slominski, Andrzej

Ślusarczyk, Sylwester

Smedley, Mark A.

Smeriglio, Antonella

Smetana, Ondrej

Smith, Mark A.

Sng, Natasha J.

Soares, Cristiano

Sobieszczuk-Nowicka, Ewa

Sogawa, Chiharu

Solanki, Manoj Kumar

Solano, Francisco

Soldatenko, Sergei A.

Solovyev, Nikolay

Soltabayeva, Aigerim

Sołtys-Kalina, Dorota

Sommaggio, Daniele

Song, Guoqing

Soriano, Jose Miguel

Sosnowska, Katarzyna

Sousa, Joana L. C.

South, Paul F.

Souza, Pedro

Sowa, Ireneusz

Spałek, Krzysztof

Španič, Valentina

Speciale, Antonio

Spiegel, Holger

Spyroglou, Gavriil

Sreedasyam, Avinash

Sroka, Zbigniew

Stacewicz-Sapuntzakis, Maria
Stadler, Marc

Stankovic, Milan

Staples, Timothy

Starfinger, Uwe

Starkuviene, Vytaute

Starns, Heath

Starowicz, Małgorzata

Stefanaki, Anastasia

Stefanou, Stefanos

Stelinski, Lukasz L.

Stevanato, Piergiorgio

Stevens, Rebecca G.

Stewart Jr., Charles

Stobiecki, Maciej

Stoleru, Vasile

Stotz, Henrik U.

Stout, Michael J.

Strati, Irini F.

Stuart, Jeff

Studnicki, Marcin

Stührwohldt, Nils

Stuper-Szablewska, Kinga

Stützel, Hartmut

Subramani, Mayavan

Subramani Paranthaman, Balasubramani

Suen, Der-Fen

Sugier, Piotr

Sullivan, Stuart

Sun, Fengiie

Sung, Jwakyung

Sung, Shi-Jean S.

Sung, Sibum

Surapathrudu, Kanakala

Suryawanshi, Vasantika

Suwazono, Yasushi

Suzaki, Takuya

Suzuki, Nobuhiro

Suzuki, Yoshihito

Swiezewska, Ewa

Sword Sayer, Mary Anne

Synowiec, Agnieszka

Syromyatnikov, Mikhail Y.

Sytar, Oksana

Szczuka, Ewa

Szepesi, Agnes

Szewczyk, Katarzyna

Szumny, Antoni

Szwengiel, Artur

Szydlowski, Nicolas

Szymanowska, Urszula

Szymanowska-Pułka, Joanna

Tabassum, Samiya

Tacchini, Massimo 
Tada, Yuichi

Taguchi-Shiobara, Fumio

Takabayashi, Junji

Takada, Yoshinobu

Takagi, Daisuke

Takahashi, Misa

Takahashi, Toshiyuki

Takano, Atsuko

Takeda, Seiji

Tamaoki, Masanori

Tanaka, Hisashi

Tang, Renjie

Tang, Yuhong

Taranto, Francesca

Tarkowská, Danuše

Tarkowski, Petr

Tate, Rothwelle J.

Tattini, Massimiliano

Tava, Aldo

Teeri, Teemu

Teh, Soon Li

Teixeira, Rita

Tekiela, Daniel

Telewski, Frank

Teresa, Del Castillo-Santaella

Terol, Javier

Terpolilli, Jason J

Tervahauta, Arja I

Terzaghi, William

Tétard-Jones, Catherine

Tetlow, Ian

Thalmann, Matthias

Thammina, Chandra Sekhar

Thérèse Charles, Marie

Thilmony, Roger

Thiruvengadam, Muthu

Thomas, Frank

Thomson, Linda

Thorwarth, Patrick

Tian, Li

Tielbörger, Katja

To, Kin-Ying

Tobias, Peri

Todaka, Daisuke

Todorova, Dessislava A.

Tokarska-Guzik, Barbara

Tokuhisa, James

Tomczyk, Michal

Tomczykowa, Monika

Tomi, Félix

Tomkowiak, Agnieszka

Tomooka, Norihiko

Topalidou, Eleni
Toriba, Taiyo

Torn, Kaire

Torra, Joel

Torricelli, Renzo

Toyota, Koiki

Trammell, Sam

Tranbarger, Timothy John

Traw, Milton Brian

Trcek, Janja

Trdan, Stanislav

Treder, Jadwiga

Trentini, Alessandro

Tripodi, Gianluca

Troitsky, Alexey

Trotta, Andrea

Trovato, Maurizio

Trumble, John

Tsakirpaloglou, Nikolaos

Tsaltas, Dimitris

Tseng, Chih-Hua

Tsitsekian, Dikran

Tubana, Brenda

Tuenter, Emmy

Tuncel, Aytug

Tundis, Rosa

Tuomivirta, Tero

Turkovskaya, Olga V.

Turner, Shane

Twardowski, Tomasz

Tyagi, Swati

Tyrka, Mirosław

Ueda, Minoru

Uesugi, Akane

Uimari, Anne

Ullah, Chhana

Umehara, Mikihisa

Upadhyay, Rakesh K.

Uprety, Yadav

Urbaniak, Jacek

Urza, Alexandra K.

Uygun, Sahra

Valentine, Tracy

Valenzuela, Juan Luis

Valkov, Vladimir

Valletta, Alessio

Vallini, Giovanni

Van den Berg, Cássio

Van Der Linde, Karina

Van Klink, John William

Van Os, Erik

Van Velzen, Ewoud J. J.

Van Wuytswinkel, Olivier

Varallyay, Eva 
Varoni, Elena Maria

Varotto, Serena

Vaughan, Martha M

Veatch-Blohm, ME

Vencill, William

Venkatesan, Thiagarajan

Venturi, Francesca

Venugopal, Mendu

Veres, Szilvia

Verger, Stéphane

Vergine, Marzia

Verstraeten, Inge

Vicario, Saverio

Vicente, Joana G.

Vicente Pérez, Rubén

Videau, Patrick

Vielba, Jesús María

Vigani, Gianpiero

Villegas, Dolors

Vincenot, Christian Ernest

Vincourt, Patrick

Vining, Kelly

Vinyard, David

Virdi, Kamaldeep

Virjamo, Virpi

Vítová, Milada

Vitti, Antonella

Vittorioso, Paola

Vodnar, Dan Cristian

Vojta, Lea

Volaire, Florence

Volkov, Vadim

Vurro, Maurizio

Vuts, Jozsef

Vyhnánek, Tomáš

Wajs-Bonikowska, Anna

Walker, Robert J.

Wanasundara, Janitha

Wang, Chao-Min

Wang, Chih-Li

Wang, Jinxiang

Wang, Peng

Wang, Sheng-Yang

Wang, Sishuo

Wang, Xutong

Wang, Yifei

Wang, Ying

Wang, Yuan

Wang, Zhenglin

Ward, John

Warner, Douglas

Waszczak, Cezary

Watanabe, Makoto
Wei, Robert

Weiss, Julia

Wen, Amy M.

Weng, Mao Lun

Weng, Xiaoyu

Weraduwage, Sarathi M.

West, Natalie M

Whitney, Cory

Widemann, Emilie

Wiersma, Andrew T.

Wiggins, Greg

Wilkinson, Mark D.

Willick, Ian

Wilmowicz, Emilia

Wimmer, Monika A.

Winkler, Daniel E

Winkworth, Richard

Winterhagen, Patrick

Wishart, Jane

Wiszniewska, Alina

Witcher, Anthony

Witte, Claus-Peter

Wittkop, Benjamin

Wojtunik-Kulesza, Karolina A.

Wojtyla, Łukasz

Wolny, Elzbieta

Woodrow, Pasqualina

Woodward, Andrew

Woznicki, Tomasz L.

$\mathrm{Wu}, \mathrm{Hao}$

$\mathrm{Wu}$, Honghong

Wu, Shan

Wullschleger, Stan D.

Wyszkowska, Jadwiga

Xavier, Alencar

Xiao, Wenyan

$\mathrm{Xu}$, Peng

$\mathrm{Xu}, \mathrm{Yi}$

Xuan, Tran Dang

Yahata, Masaki

Yahr, Rebecca

Yamagata, Yoshiyuki

Yamakawa, Takeo

Yamasaki, Hideo

Yan, Mary

Yandeau-Nelson, Marna D.

Yang, Gaowen

Yang, Peiqi

Yang, Seung Hwan

Yang, Shun-Chin

Yang, Yu-Chiao

Ye, Liyun

Yeh, Ching-Hui 
Yeung, Elaine

Ying, Sheng

Yokosyo, Kengo

Yokota, Shinso

Yoshida, Kaoru T.

Yoshida, Keisuke

You, Chenjiang

You, Frank M.

$\mathrm{Yu}$, Xiang

$\mathrm{Yu}$, Xingwang

Yuann, Jeu-Ming P.

Zabetakis, Ioannis

Zacchini, Massimo

Zahoranova, Anna

Zaidi, Syed Shan-E-Ali

Zaini, Paulo

Zaitlin, David

Zakrzewski, Jerzy

Zamboni, Anita

Zamyatnin, Andrey

Zancani, Marco

Zapata Coll, Pedro Javier

Zapata-Sierra, Antonio

Zarabska-Bożejewicz, Daria

Zarrelli, Armando

Zarrouk, Olfa

Zell, Roland

Zeller, Alexander K.

Zhai, Zhiyang

Zhang, Cankui

Zhang, Fei
Zhang, Jin

Zhang, Junli

Zhang, Wei

Zhang, Wenheng

Zhao, Cheng

Zhao, Dongyan

Zhao, Jianfei

Zhao, Jinping

Zhou, Bangjun

Zhou, LinLi

Zhou, Rong

Zhou, Zhenjiang

Zhu, Changfu

Zhu, Yingjie

Žiarovská, Jana

Zielińska, Sylwia

Zimmermann, Sabine Dagmar

Ziogas, Vasileios

Zitko, Jan

Zivcak, Marek

Zovko Končić, Marijana

Źróbek-Sokolnik, Anna

Zub, Karol

Zubieta, Chloe

Zuk, Magdalena

Zuluaga, Paola

Żurek, Grzegorz

Zuriaga, Elena

Zyprian, Eva 